\title{
Quantification of Brownian motion under presence of flow using Particle Diffusometry
}

\author{
D. Lee ${ }^{1}$, S. T. Wereley ${ }^{1 *}$ \\ ${ }^{1}$ Purdue University, School of Mechanical Engineering, West Lafayette, USA \\ *wereley@purdue.edu
}

\begin{abstract}
Particle diffusometry (PD), a quantification method for the Brownian motion, is performed by recording temporally sequential images and using correlation analysis to obtain an ensemble diffusion coefficient for all particles captured in the imaging region (Clayton et al., 2017). PD is proven to be successful in the detection of the waterborne pathogen $V$. cholerae in environmental samples using different imaging techniques, including an inverted fluorescence microscope as well as a handheld hardware device operated with a smartphone (Clayton et al., 2019; Moehling et al., 2020). Although we intend to use PD to calculate diffusion coefficients in quiescent fluid, oftentimes unintentional fluid flows occur, creating measurement error when calculating the diffusion coefficient. In previous work, recordings under the presence of flow were discarded to avoid incorrect measurements of the sample. The diffusion coefficient is calculated as:

$$
D=\frac{S_{c}^{2}-S_{a}^{2}}{16 M^{2} \Delta t}
$$

Experimentally recorded image frames are obtained at an inter-frame time $\Delta \mathrm{t}$. These frames are then parsed into interrogation windows. Interrogation windows from an image taken at $\Delta \mathrm{t}$ is correlated with itself to obtain autocorrelation peak width $S_{a}$. Two separate image frames recorded at $\mathrm{t}$ and $\mathrm{t}+\Delta \mathrm{t}$ are correlated to obtain cross-correlation peak width $S_{c}$. The $S_{c}$ broadens compared to the $S_{a}$ due to the presence of Brownian motion. The obtained peak widths can be then averaged spatially or temporally over number of image frames, to lower the random uncertainty of the measurement. The diffusion coefficient is then calculated using Eq. 1 (Olsen and Adrian, 2000), where $\mathrm{M}$ is the total magnification of the recording setup. In this body of work, we modify our approach in measuring the diffusion coefficient in the presence of fluid flow.
\end{abstract}

In the absence of flow, the correlation peaks described above produce axisymmetric Gaussian profiles. However, in the presence of flow, the correlation peak center shifts to the direction of the flow-exactly the principle behind PIV. If the flow also has gradients in it, the correlation peak stretches and rotates in the direction of shear, no longer axisymmetric. Since the Brownian motion along each axis is independent of others, proper identification of streamwise and cross-streamwise directions allow a generalized rotated two-dimensional elliptical Gaussian function to be fit to the cross-correlation (Chamarthy et al., 2009). The diffusion coefficient can be calculated from the cross-streamwise correlation peak width.

In this work, simulated Brownian motion images were generated with a frame rate of 15 frames per second with a diffusion coefficient of $1 \times 10^{-12} \mathrm{~m}^{2} / \mathrm{s}$ with a variety of conditions: 0 to 80 degrees rotation with 10 -degree increments, 1 to $10 \mathrm{pixel} / \Delta \mathrm{t}$ flow velocity with $10 \mathrm{pixel} / \Delta \mathrm{t}$ increments, and three different flow types (uniform, Couette, and Poiseuille flows). Once the correlation peaks were calculated, all the peaks from each of the interrogation windows were averaged temporally over 100 frames image sets to reduce statistical random errors. A 3-point Gaussian peak fit was applied in both $\mathrm{x}$ - and y-directions to determine the sub-pixel maximum location for further flow analysis. To calculate the cross-streamwise peak width, a total of $11 \times 11 \mathrm{pixel}^{2}$ areas were fitted (in contrast to the typical 5-point PIV peak fit), and the ellipse equations at $e^{-1}$ height is calculated using the fitted Gaussian profile. Resulting diffusion coefficient values of varying angles and velocities were averaged with respect to the induced velocity values.

As shown in Fig. 1(a) a 3-point Gaussian sub-pixel fit on a single pass correlation poses no difficulty in estimating the flow velocity from the generated simulation videos. A larger measurement variation occurred 
in the low flow velocity region (i.e. 1-4 pixel/s), where distinguishing between the flow velocity and the Brownian motion of particles presents a greater margin of error. For the case of measuring particle diffusion coefficient, presented in Fig. 1(b), the diffusion coefficient calculated in the presence of uniform flow show minimal deviation from their simulated values, hence the smaller error. However, with non-uniform flows, we see greater deviations from theoretical values due to the gradient of the flow. Overall, an increase in error is observed as the flow velocity is increased. Analysis reveals that with the developed algorithm, analyzed diffusion coefficients show an error greater than $10 \%$ when the flow velocity exceeds $8 \mathrm{pixel} / \Delta \mathrm{t}$.

Experimentally, flows were generated using a syringe pump through a rectangular channel. As the flow profile forms an elongated Poiseuille flow along the wider dimension of the channel, the focal point is adjusted to ensure the recorded flow profile is that of a uniform flow. Simulation parameter for the uniform flow is extended to $22 \mathrm{pixel} / \Delta \mathrm{t}$ to better compare the trend between the simulation and experiment results. Due to the difficulty of obtaining even increment of volumetric flow, $\mathrm{x}$-directional error bar is incorporated to account for the variation. The resulting Fig. 1(c) shows the experimental data following the trend of the simulation results. The experiment shows the analyzed diffusion coefficients results in an error greater than $10 \%$ when the flow exceeds 18 pixel $\Delta \mathrm{t}$ with the developed algorithm. These 18 pixels account for $17 \%$ of overall interrogation window. In other words, the velocity is equivalent to $6.6 \mu \mathrm{m} / \Delta \mathrm{t}$, a significant movement if we take into account the particle size of $470 \mathrm{~nm}$ that is used for the experimental verification. This current body of work shows a promising initial work toward measuring diffusion coefficients in the presence of fluid flow.

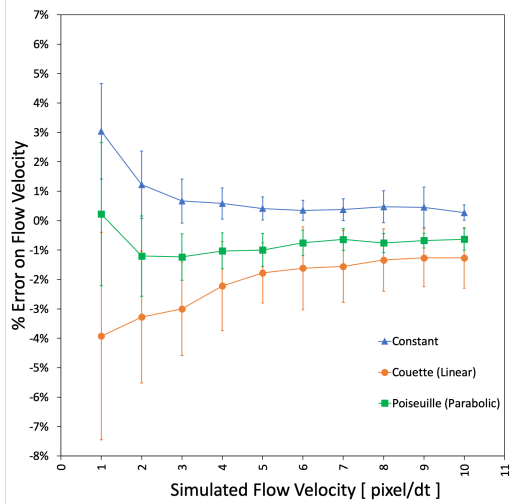

(a) Flow Velocity

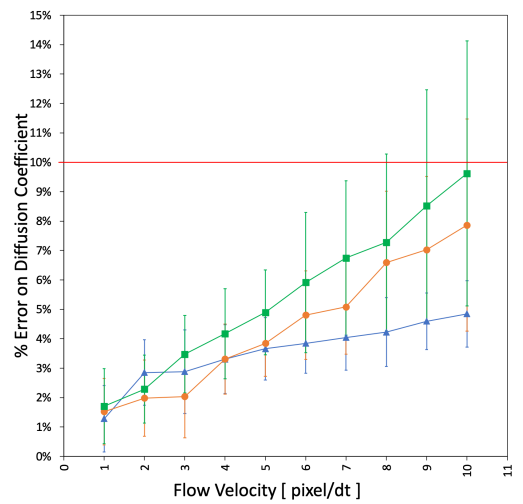

(b) Diffusion Coefficient

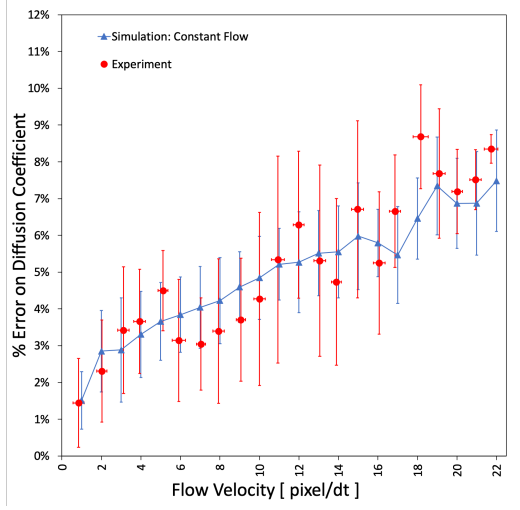

(c) Experimental Verification

Figure 1: Comparison of simulation results with analyzed values (a,b), and with experiment values (c).

\section{References}

Chamarthy P, Garimella SV, and Wereley ST (2009) Non-intrusive temperature measurement using microscale visualization techniques. Experiments in Fluids 47:159-170

Clayton KN, Lee D, Wereley ST, and Kinzer-Ursem TL (2017) Measuring biotherapeutic viscosity and degradation on-chip with particle diffusometry. Lab Chip 17:4148-4159

Clayton KN, Moehling TJ, Lee D, Wereley ST, Linnes JC, and Kinzer-Ursem TL (2019) Particle Diffusometry: An Optical Detection Method for Vibrio cholerae Presence in Environmental Water Samples. Scientific Reports 9:1739

Moehling TJ, Lee D, Henderson ME, McDonald MK, Tsang PH, Kaakeh S, Kim ES, Wereley ST, KinzerUrsem TL, Clayton KN, and Linnes JC (2020) A smartphone-based particle diffusometry platform for sub-attomolar detection of vibrio cholerae in environmental water. Biosensors \& Bioelectronics $167: 112497$

Olsen MG and Adrian RJ (2000) Brownian motion and correlation in particle image velocimetry. Optics \& Laser Technology 32:621-627. optical methods in heat and fluid flow 